\title{
Effect of Systemic Therapies on Outcomes following Vertebroplasty among Patients with Multiple Myeloma
}

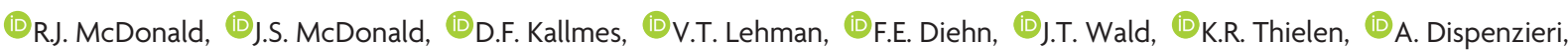
and $($ P.H. Luetmer

\begin{abstract}
BACKGROUND AND PURPOSE: The role of vertebroplasty in patients with myeloma remains relatively undefined. Accordingly, we sought to better define the efficacy of vertebroplasty for myeloma-associated fractures and determine the effect of procedure timing relative to the initiation of systemic therapy on outcomes and complication rates.
\end{abstract}

MATERIALS AND METHODS: Clinical, laboratory, and medication data were retrieved for 172 patients with multiple myeloma treated with vertebroplasty since October 2000. Quantitative outcome data (Roland-Morris Disability Questionnaire [scale, 0-24] and the Numeric Rating Scale [0-10] for pain at rest and with activity) were collected immediately pre- and postoperatively and at 1 week, 1 month, 6 months, and 1 year following vertebroplasty. Patients with $\geq 50 \%$ improvement on the Numeric Rating Scale and $\geq 40 \%$ improvement on the Roland-Morris Disability Questionnaire were classified as "responders." Peri- and postoperative complications were also collected.

RESULTS: Significant median improvement in the Roland-Morris Disability and rest and activity Numeric Rating Scale scores (15, 2, and 6 points, respectively; $P<.0001)$ persisted at 1 year without significant change from the immediate postoperative scores $(P>.36)$. Patients on systemic therapy at the time of vertebroplasty were more likely to achieve "responder status," compared with patients not on systemic therapy, for the Numeric Rating Scale pain at rest score $(P<.01)$ and the Roland-Morris Disability Questionnaire score $(P<.003)$, with no difference in complication rates $\left(\chi^{2}=0.17, P=.68\right)$.

CONCLUSIONS: Vertebroplasty is an effective therapy for patients with myeloma with symptomatic compression fractures. Favorable outcomes are more likely to be achieved when spinal augmentation is performed after systemic therapy is initiated. Complication rates were not affected by the timing of systemic therapy.

ABBREVIATIONS: IQR = interquartile range; NRS = Numeric Rating Scale; RDQ = Roland-Morris Disability Questionnaire

$F^{\prime}$ or the past 20 years, vertebroplasty has been shown to be an effective treatment for symptomatic vertebral compression fractures refractory to medical therapy. ${ }^{1,2}$ Although recent evidence suggests that the pain reduction derived from this procedure may not be attributable to the injection of the cement itself, the data are clear that vertebroplasty recipients experience durable improvement in mobility and reduced narcotic use that persist for months to years following therapy. ${ }^{3}$ Although patients with osteoporosis comprise most vertebroplasty recipients in the United States, traumatic and pathologic fractures have also

Received April 27, 2016; accepted after revision July 8.

From the Departments of Radiology (R.J.M., J.S.M, D.F.K., V.T.L., F.E.D., J.T.W., K.R.T., P.H.L.), Neurosurgery (D.F.K.), and Hematology (A.D.), College of Medicine, Mayo Clinic, Rochester, Minnesota.

Please address correspondence to Robert J. McDonald, MD, PhD, Department of Radiology, Mayo Clinic, 200 1st St SW, Rochester, MN 55905; e-mail: mcdonald. robert@mayo.edu

http://dx.doi.org/10.3174/ajnr.A4925 been treated with percutaneous spinal augmentation. ${ }^{4-6}$ Among pathologic fracture etiologies, multiple myeloma is one of the more common indications for intervention. Patients with myeloma are particularly prone to pathologic vertebral compression fractures due to systemic osteoporosis from cytokine-mediated imbalance of osteoclast and osteoblast function and systemic corticosteroid therapy. ${ }^{6}$

Although a large body of evidence exists demonstrating the efficacy of vertebroplasty among patients with benign osteoporotic vertebral compression fractures, the data in support of its use in the treatment of pathologic fractures among patients with multiple myeloma remain limited. In part, data from patients with myeloma are limited due to their reduced survival time, clinical uncertainty with respect to improvement in pain in the setting of diffuse disease, uncertainty as to when to treat, and a higher threshold of treatment criteria for patients with diffuse disease. In contradistinction to patients with benign compression fractures, patients with myeloma are often on multidrug systemic therapy 
and may have hematologic derangements related to their disease and/or treatment. These factors may confound outcomes and potentially render patients with myeloma more predisposed to adverse events.

The purpose of this study was to better define the efficacy of vertebroplasty for myeloma-associated fractures and determine the effect of procedure timing relative to the initiation of systemic chemotherapy and the extent of disease on outcomes and complication rates.

\section{MATERIALS AND METHODS Study Design and Population}

Design and execution of this single-center retrospective study were approved by our institutional review board and complied with Health Insurance Portability and Accountability Act privacy guidelines. Patients were included in this study if they met the following 3 criteria: 1) They had an established diagnosis of multiple myeloma or myeloma-related disease (plasmacytoma or plasma cell proliferative disease) within 30 days of spine augmentation, 2) demonstrated MR imaging evidence of a vertebral compression fracture, and 3) were evaluated and subsequently underwent percutaneous vertebroplasty at our medical center. Patients who underwent ablation during their augmentation, lacked radiographic evidence of compression fracture, experienced improvement in clinical pain syndrome with conservative management, had technical contraindications to vertebroplasty, or had noncorrelating pain were excluded from enrollment in our longitudinal vertebroplasty patient data base Although rarely treated in our practice, large symptomatic lytic lesions without radiographic evidence of vertebral body fracture were not included in this study cohort. Among patients who underwent multiple vertebroplasty procedures across time, data were limited to the first vertebroplasty procedure to mitigate potential confounding effects of recurrent fractures on treatment outcomes. Approximately $40 \%$ of patients in this study were included in a previous publication that did not specifically examine the effects of systemic therapy timing on outcomes. ${ }^{6}$

\section{Clinical Evaluation}

Multiple myeloma or myeloma-related disease was diagnosed by using standard clinical, laboratory, and imaging criteria. ${ }^{7}$ In patients with multiple fractures, clinical assessment of painful spinal levels included collection of clinical history, physical examination, and imaging. Clinical findings of pain on palpation or imaging evidence of intervertebral marrow edema with MR imaging or both were used to guide treatment-level selection.

\section{Radiologic Evaluation}

Radiographic evaluation of myeloma-related fractures consisted primarily of MR imaging supplemented with CT and/or bone radiographs. MR imaging examinations of patients meeting all inclusion and exclusion criteria were re-evaluated by 5 neuroradiologists (V.T.L., F.E.D., J.T.W., K.R.T., P.H.L.) with 3, 6, 19, 19, and 24 years of respective experience who were blinded to clinical data and patient outcomes. MR imaging studies included appropriate spin-echo enhancement and inversion recovery suppres- sion techniques to increase the conspicuity of malignant fractures and ascertain the extent of spinal disease.

\section{MR Imaging Classification of Spinal Lesions and Involvement}

In an effort to characterize vertebroplasty-treated compression fractures in the myeloma population, preoperative MR images of treated fractures were grouped into 3 categories, as previously described $^{6}$ : Type 1 compression fractures had no MR imaging evidence of myeloma involvement and were classified by using the following morphologies: normal-appearing fatty marrow in some portion of the vertebral body with no evidence of pedicle involvement or epidural or paraspinal disease; and/or absence of focal or diffusely abnormal marrow signal intensity within the treated vertebral body. Type 2 compression fractures were considered indeterminate for underlying myelomatous disease and were classified by diffusely low T1 marrow signal intensity of the treated vertebral body and absence of paraspinal disease. Type 3 compression fractures had definitive MR imaging evidence of myelomatous disease within the vertebral body with additional involvement of the pedicles, paraspinal tissue, and/or epidural tissue. The appearance of adjacent marrow remote from the treated vertebrae had no impact on fracture characterization.

The extent of myelomatous disease in the untreated thoracic and lumbar vertebral bodies was characterized independently from the treated vertebra as previously described ${ }^{6}$ : grade I, normal appearance of the remainder of the visualized spine; grade II, 1 or several focal vertebral lesions consistent with myeloma $(<10$ lesions involving the thoracic and lumbar spine or $<6$ lesions involving either the thoracic or lumbar spine); or grade III, diffuse vertebral bone marrow involvement ( $\geq 10$ lesions involving the thoracic and lumbar spine or $\geq 6$ lesions involving either thoracic or lumbar spine).

\section{Vertebroplasty Procedure}

Vertebroplasties were performed by 9 experienced staff radiologists as previously described. ${ }^{1,7,8}$ Periprocedural pain management included a combination of intravenous conscious sedation and local anesthesia of the soft tissues superficial to the targeted spinous pedicles. Biplanar fluoroscopy was used to monitor needle position, trajectory, and intraosseous injection of polymethylmethacrylate. After needle position was confirmed, polymethylmethacrylate was freshly prepared by mixing barium sulfatedoped solid copolymer (methylmethacrylate) with a small amount of liquid initiator (N,N-dimethyl para-toluidine) and subsequently injected into the compressed vertebral body. Cement injection was terminated when there was adequate filling of the vertebral body (typically $1-4 \mathrm{~mL}$ of polymethylmethacrylate) or fluoroscopic evidence of epidural, venous, or transendplate extravasation or extrusion of cement into the posterior quarter of the vertebral body. Following vertebroplasty, patients were discharged after 2 hours of strict bed rest and a postprocedural visit from the performing radiologist.

\section{Data Sources}

Vertebroplasty data (dates of diagnosis, treatment, approximate onset of symptoms, procedural data and notes, and radiologic

AJNR Am J Neuroradiol 37:2400-06 Dec 2016 www.ajnr.org

2401 
studies), laboratory data, and pharmacy data were obtained from archived institutional electronic medical records. Vertebroplasty outcome data were retrieved from our institutional electronic data base used to monitor safety and efficacy; this data base contains data from approximately 2350 patients treated with vertebroplasty at our medical center since 1999.

\section{Outcome Measures}

Patient outcome scores were collected by trained nursing staff in person immediately before and after vertebroplasty and via telephone 1 week, 1 month, 6 months, and 1 year following vertebroplasty. Quantitative patient outcomes included the Numeric Rating Scale (NRS) of pain at rest and pain with activity scored on a $0-10$ scale and the Deyo-modified Roland-Morris Disability Questionnaire (RDQ) scored on a 0- to 24-point scale. ${ }^{9}$ Outcomes were calculated as median values with interquartile ranges and percentage changes for each time point and patient group. Percentage changes from the preoperative baseline were calculated for the NRS and RDQ scores for each patient at each time point; patients who attained $\geq 50 \%$ improvement in NRS and $\geq 40 \%$ improvement in RDQ scores were classified as "responders," patients with $<50 \%$ improvement in NRS and $<40 \%$ improvement in RDQ scores were classified as "suboptimal responders," and patients with no change or worsening of scores were classified as "nonresponders" based on standard definitions. ${ }^{10}$ After 3 failed attempts, patients who could not be reached by phone were designated as "nonreporter status" for that specific time point; nonreporter status did not preclude telephone contact at a later time unless the patient formally withdrew from further study participation.

\section{Statistical Analysis}

All statistical analyses were performed by using R statistical and computing software (http://www.r-project.org/) and JMP, Version 10 (SAS Institute, Cary, North Carolina). Continuous data were displayed as median scores with interquartile ranges due to non-normal distributions and were compared by using the Wilcoxon signed rank test. Categoric data were displayed as relative frequencies (percentages) and were compared by using $\chi^{2}$ tests of significance. Comparisons of means of continuous datasets between $\geq 2$ categoric groups were performed by using analysis of variance. Nonparametric pair-wise correlations between continuous data were performed by using the Wilcoxon rank sum test. Significance was assigned to differences of $P \leq .05$.

\section{RESULTS}

\section{Patient Population}

Among 2350 patients treated with vertebroplasty between October 2000 and December 2014, 181 (8\%) had a diagnosis of myeloma or myeloma-related disease. One hundred seventy-two of the 181 patients had undergone preprocedural MR imaging (cervical spine MR imaging examinations, $n=21$; thoracic spine MR imaging examinations, $n=108$; lumbar spine MR imaging examinations, $n=82$ ) and were included for further study. Sixty-seven (39\%) of these patients with myeloma were included in a previous publication detailing our initial vertebroplasty experience in this patient subpopulation that did not address the timing of systemic

\begin{tabular}{|c|c|c|}
\hline Variable & $\begin{array}{l}\text { Treated Group } \\
\quad(n=134)\end{array}$ & $\begin{array}{l}\text { Untreated Group } \\
\qquad(n=38)\end{array}$ \\
\hline Male sex (No.) (\%) & $74(55 \%)$ & $23(61 \%)$ \\
\hline Deceased at $1 \mathrm{yr}$ & $19(14 \%)$ & $4(11 \%)$ \\
\hline Duration of pain (mo) & $3(1-7)$ & $2(1-3)$ \\
\hline Delay in diagnosis (mo) & $9.5(2.5-45)$ & NA \\
\hline No. of affected levels & $5(3-10)$ & $5(2-12)$ \\
\hline No. of treated levels & $2(1-3)$ & $2(1-3)$ \\
\hline
\end{tabular}

Note:-NA indicates not applicable.

${ }^{a}$ Data are median (IQR) unless otherwise indicated.

therapy on clinical outcomes or adverse events. ${ }^{6}$ Among the treated patients with myeloma, 134 (76\%) were on systemic drug therapy at the time of vertebroplasty, while the remaining 38 (24\%) either were recently diagnosed or initially presented with vertebral compression fracture as the first symptomatic manifestation of multiple myeloma. The clinical and demographic characteristics of these groups are shown in the Table.

\section{Treated and Affected Levels}

Vertebroplasty was performed on 287 vertebral levels in the 172 patients in our study group. The distribution of spinal levels treated and affected by multiple myeloma is shown in Fig $1 A,-B$, respectively. In our study population, the prevalence of fractures followed a bimodal distribution, being most common at T7/T8 and T12/L1. More diffuse cases of spine involvement were noted in 18 patients, representing $10 \%$ of all patients included in this study. Ten patients included in this study had concomitant cervical spinal fractures; vertebroplasty was never performed on these cervical fractures due to technical reasons and the presence of concomitant symptomatic fractures at lower load-bearing vertebral levels. Within this study cohort, the median number of fractured vertebrae (4; interquartile range [IQR], 2-5) was significantly greater than the median number of treated vertebrae (2; IQR, 1-3) per patient $(P=.001)$. The median number of untreated, presumably asymptomatic, vertebral compression fractures was 2.5 (IQR, 1-4) per patient. No significant differences in the number of affected or treated levels per patient were identified between male and female patients (affected level: $\chi^{2}=0.48, P=$ .49 ; treated level: $\chi^{2}=0.17, P=.68$ ).

Among the patients in the study population, 64 (37\%) had diffuse (grade III) disease at the time of vertebroplasty, while the remaining 108 patients (63\%) had more localized (grade I or II) disease limited to $<10$ thoracic and lumbar vertebral bodies. Of the 287 treated vertebral fractures, 138 (48\%) had no MR imaging evidence of myelomatous involvement (type 1), $71(25 \%)$ had indeterminate myelomatous involvement (type 2 ), and 78 (27\%) had definite MR imaging evidence of pathologic vertebral compression fracture (type 3 ). Representative examples of each compression fracture type are shown in Fig 2.

\section{Time to Treatment}

Among the 172 patients with myeloma, 38 (22\%) presented with a painful compression fracture as the first evidence of multiple myeloma, while the remaining 134 (78\%) had a diagnosis of myeloma before the discovery of a fracture. Within the subset of patients who initially presented with vertebral fracture, systemic 


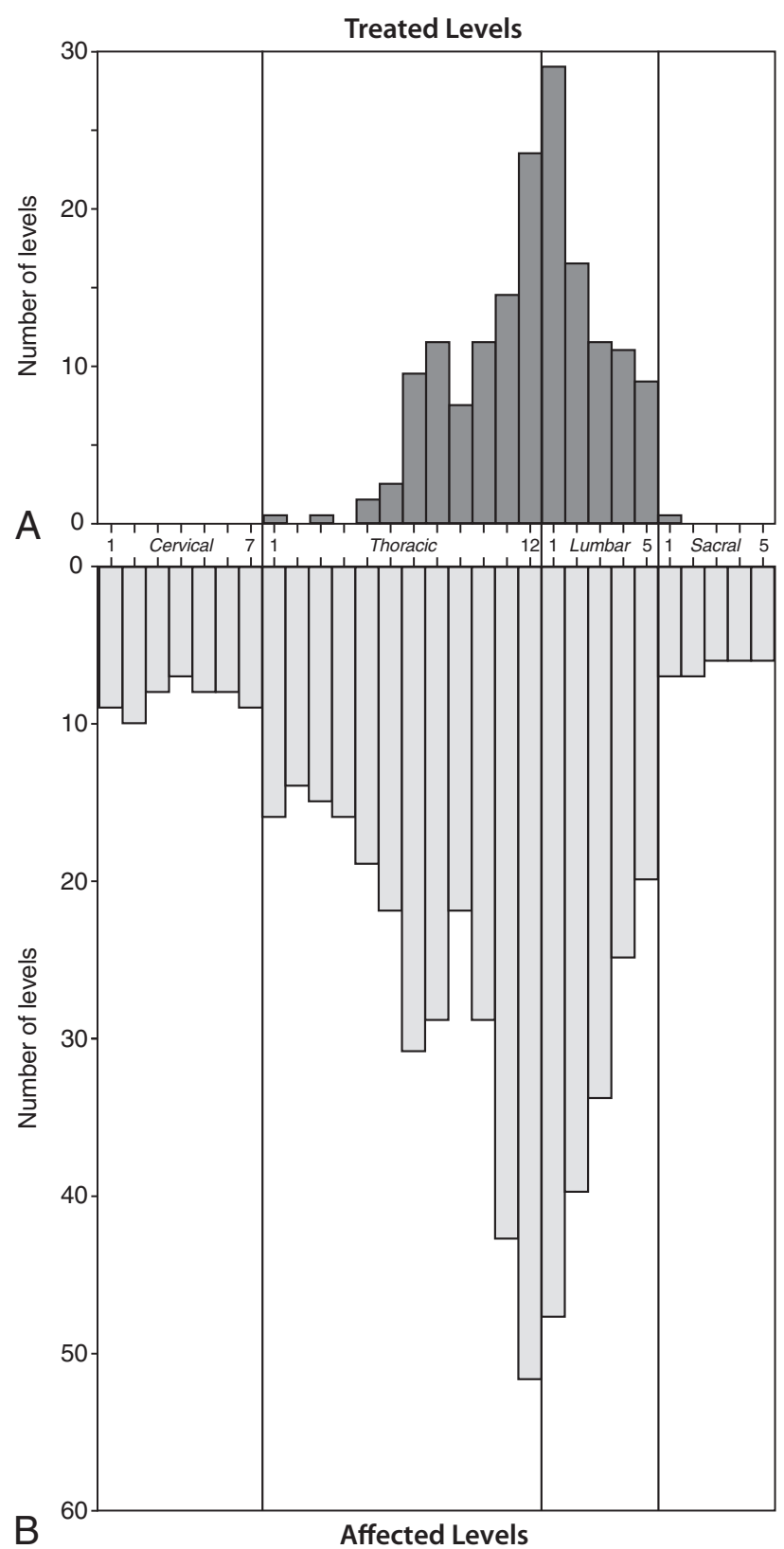

FIG 1. Treated and affected levels. Vertebral level frequency histograms of 287 treated vertebral levels $(A)$ and 493 affected vertebral levels $(B)$ in the 172 patients comprising the study population of patients with myeloma.

drug therapy was initiated in $2(5 \%)$ patients before vertebroplasty, and these systemic therapies began within several days of vertebroplasty. Among the remaining 134 patients with a diagnosis of myeloma at the time of vertebroplasty, 111 (82\%) were on systemic therapy before vertebroplasty, while the remaining 23 $(18 \%)$ were treated for myeloma after vertebroplasty. The median time between the diagnosis of myeloma and the initiation of systemic drug therapy was 10 months, with a range of 1-279 months (IQR $=3-45$ months) (Fig 3A). Seventy-one (53\%) of these patients underwent vertebroplasty within 12 months of the initial diagnosis. The median time between the onset of symptoms (back pain with subsequent radiologic evidence of compression fracture) to systemic treatment was 3 months, with a range of $0-84$ months (IQR $=1-7$ months) (Fig 3B).

\section{Clinical Outcomes}

Quantitative pain and disability data were collected postoperatively on all 172 patients. To date, 148 (86\%) patients had quantitative (NRS pain at rest/activity and RDQ) outcome data collected at the 1-month end point; 135 (78\%), at the 6-month end point; and 111 (65\%), at the 1-year end point. Of the 61 patients missing follow-up data at the 1 -year end point following vertebroplasty, 26 (43\%) had an intervening vertebroplasty procedure ( $<1$ year ago), $12(20 \%)$ were lost to follow-up for unspecified reasons, and $23(38 \%)$ died. The relatively high mortality rate in our cohort can be attributed to the more enriched pool of patients with advanced and refractory disease that seek care at our quaternary care center; on review of the medical records, none of these deaths were directly attributable to vertebroplasty therapy.

\section{Clinical Outcome Analysis}

For the entire study population, median preoperative quantitative outcome scores (NRS pain at rest, NRS pain with activity, RDQ score) were 4 (IQR $=2-6), 9(\mathrm{IQR}=7-10)$, and 20 (IQR = 17-22), respectively (Fig 4A). All 3 quantitative outcome measures of pain and disability demonstrated significant decreases in the postprocedural setting $(P<.0001)$. NRS data for pain at rest significantly improved in the postprocedural period following vertebroplasty $(P<.0001)$, with a median improvement of 2 points at 1 week, with no significant intervening change in scores at 1 month, 6 months, and 1 year $(P=.36-.99)$. The NRS data for pain with activity significantly improved in the postprocedural period following vertebroplasty $(P<.0001)$, with a median improvement of 6 points at 1 week with no significant intervening change in scores at 1 month, 6 months, and 1 year $(P=.41-.99)$. RDQ scores demonstrated a significant median improvement of 15 points 1 week after vertebroplasty, with additional nonsignificant improvement at 6 months and 1 year following spinal augmentation $(P=.61-.99)$. There was no significant difference in the magnitude of response among the cohort of patients with loss to follow-up due to subsequent vertebroplasty and those patients who were followed for the entire study duration (NRS pain at rest, $P=.57$; NRS pain with activity, $P=.32$; RDQ score, $P=.49$ ).

For NRS pain outcomes, at least $47 \%$ and $44 \%$ of patients met the criteria for responder status ( $\geq 50 \%$ improvement in pain) for pain at rest and pain with activity in the immediate postoperative setting, respectively (Fig 4B). In general, the total fraction of patients meeting responder and/or suboptimal responder status increased with time (NRS pain with rest, $67 \%-81 \%$; NRS pain with activity, 78\%-100\%). Likewise, for RDQ outcomes, at least $40 \%$ of patients met the criteria for responder status ( $\geq 40 \%$ improvement in the RDQ score), with a large fraction of total favorable cases meeting suboptimal responder status $(0 \%-67 \%)$. The relative fraction of responders also increased with time. Furthermore, there was no significant difference in outcomes between patients who had more acute back pain ( $\leq 6$ months of pain) and those with more chronic back pain ( $>6$ months of pain) for pain at rest $(P=.28-.74)$, pain with activity $(P=.06-.90)$, and the RDQ score $(P=.22-.93)$ at any postoperative time point.

AJNR Am J Neuroradiol 37:2400-06 Dec 2016 www.ajnr.org 


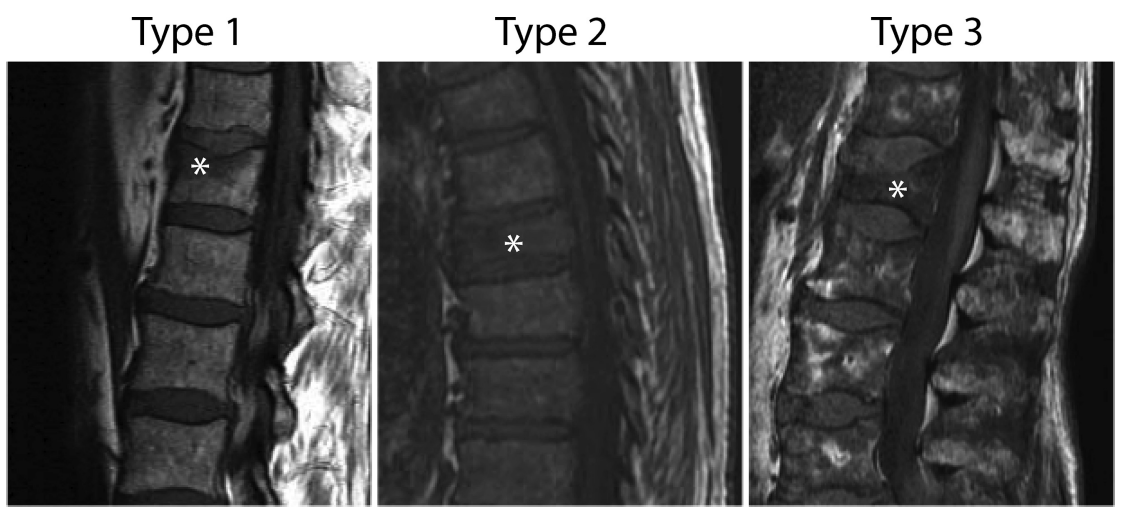

FIG 2. Sagittal TI MR images demonstrating fracture types. Stars indicate treated fractures. $A$, Type 1 fracture, considered likely osteoporotic. Areas of preserved, high T1 signal within the fractured vertebral body, without a focal intravertebral lesion and no evidence of pedicle involvement or epidural or paraspinal disease (not shown). B, Type 2 fracture, indeterminate for underlying lesions. Diffusely low-signal marrow throughout the spine. No focal lesion or epidural or paraspinal lesion within the treated vertebral body. C, Type 3 fracture, with clear evidence of a myelomatous lesion within the treated vertebral body.

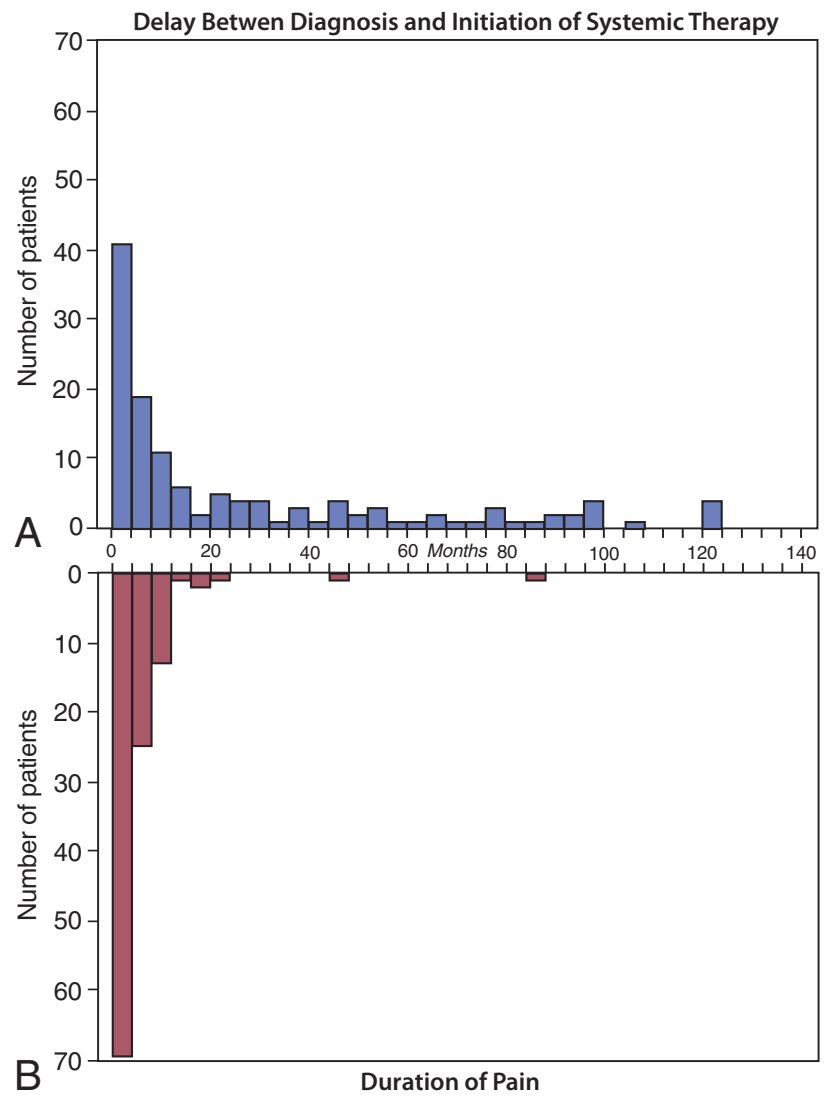

FIG 3. Diagnostic interval and duration of pain. The time interval between the diagnosis and initiation of systemic therapy $(A)$ and the duration of pain $(B)$ are shown as frequency histograms with each bin representing a 4-month interval.

\section{Effects of Systemic Treatment on Outcomes}

In an effort to determine whether systemic drug therapy affected clinical outcomes, we compared clinical outcomes and complication rates between untreated and treated patient groups. Compared with the group not treated with systemic drug therapy at the time of vertebroplasty, a significant favorable treatment effect was observed for the treated group having pain with activity $(F=8.97$,
$P=.030)$ but not with RDQ $(F=0.02$, $P=.89)$ or pain at rest $(F=1.01, P=$ $.22)$ scores. Furthermore, there were significant differences in the response across time between the untreated and treated groups having pain with activity $(F=10.14, P=.025)$ and RDQ $(F=$ 6.16, $P=.029)$ scores, but again not with pain at rest $(F=1.92, P=.78)$.

Among categoric outcomes, there was no significant difference between the treated and untreated groups at any time point for NRS pain at rest $(P=.22$ .96) (Fig 4B). Among the NRS painwith-activity categoric outcomes, a significantly higher fraction of favorable responders were present in the systemic treatment group at 1 and 6 months following vertebroplasty $(P<.01)$; a borderline significant finding was also observed for NRS pain-withactivity scores in the immediate postoperative setting $(P=.059)$. For RDQ outcomes, the systemic treatment group also had significantly higher numbers of favorable outcomes at 1 week, 1 month, and 1 year following vertebroplasty compared with the untreated group $(P<.003)$.

\section{Complications}

Forty-one (24\%) patients experienced some form of clinically asymptomatic periprocedural complication; these complications included 15 cases of inadvertent disc-space extravasation, 14 cases of inadvertent paravertebral-space extravasation, and 12 cases of cement extravasation into the epidural space without clinical sequelae. No symptomatic complications were observed in this patient cohort, and the complications did not confound further assessment of clinical status. The median white blood cell count was $4.3 \times 10^{9}$ cells $/ \mathrm{L}\left(\mathrm{IQR}=3.4-5.6 \times 10^{9}\right.$ cells $\left./ \mathrm{L}\right)$, while the platelet count was $154 \times 10^{9} / \mathrm{L}\left(\mathrm{IQR}=76-215 \times 10^{9} / \mathrm{L}\right)$. The lowest white blood cell count and platelet count at the time of vertebroplasty were $1.5 \times 10^{9}$ cells/L and $41 \times 10^{9} / \mathrm{L}$, respectively. Complications were not significantly affected by the presence of ongoing systemic therapy $\left(\chi^{2}=0.17, P=.68\right)$ or derangement in laboratory values at the time of vertebroplasty $(P>.79)$.

\section{DISCUSSION}

The findings from this single-center, retrospective study provide robust evidence that vertebroplasty confers significant and durable improvement in pain and disability among patients with myeloma with symptomatic vertebral compression fractures. Our findings suggest that vertebroplasty may be more efficacious among patients with multiple myeloma already on systemic therapy compared with those who undergo vertebroplasty before or at the initiation of therapy. Improvement in pain and disability was similar between patients with more acute and chronic durations of fracture-related pain. Furthermore, complications, when present, were clinically asymptomatic, with no known long-term sequelae and were not correlated with the timing of systemic therapy or derangement in blood chemistries; this result suggests that 

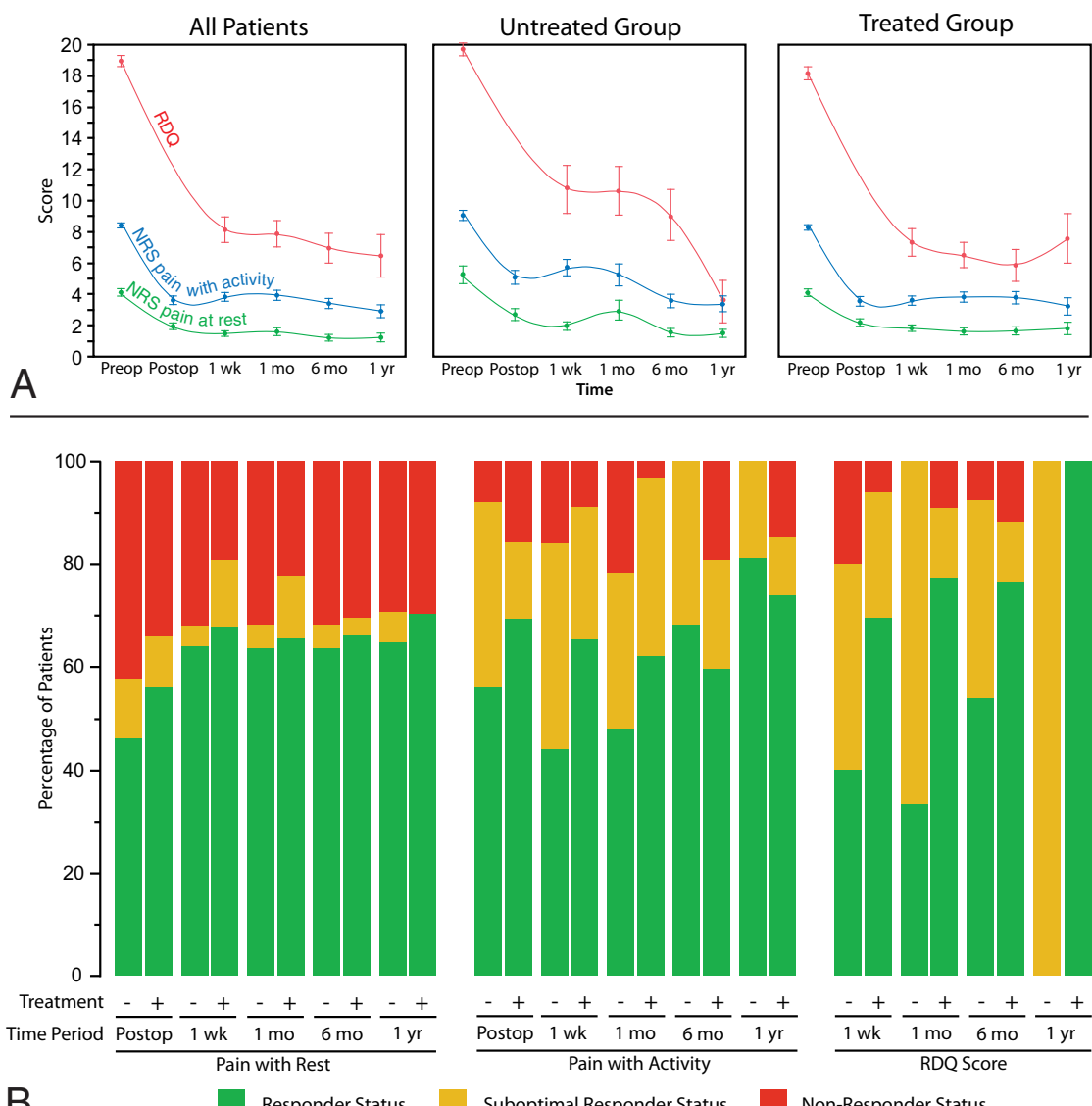

$\frac{\frac{-+}{\text { Postop }} \frac{-+}{1 \mathrm{wk}} \frac{-+}{1 \mathrm{mo}} \frac{-+}{6 \mathrm{mo}} \frac{-+}{1 \mathrm{yr}}}{\text { Pain with Activity }}$

Suboptimal Responder Status

Non-Responder Status

FIG 4. Clinical outcomes. Change in median $(A)$ and categoric $(B)$ clinical outcome scores of pain at rest, pain with activity, and RDQ scores. Median scores are shown at preoperative baseline (preop); postoperatively (postop); and 1 week, 1 month, 6 months, and 1 year following vertebroplasty for the entire study cohort (all patients), patients not on systemic therapy at the time of vertebroplasty (untreated group) and patients on systemic therapy at the time of vertebroplasty (treated group). Median RDQ scores are shown in red; NRS pain with activity, in blue; and NRS pain at rest, in green. Categoric clinical outcomes are represented as changes in pain with rest; pain with activity; RDQ scores relative to the preoperative baseline in the immediate postoperative setting (postop); and 1 week, 1 month, 6 months, and 1 year following vertebroplasty. The fraction of patients meeting criteria for responder status are shown in green; suboptimal responder status, in yellow; and nonresponder, status in red.

the safety of vertebroplasty in the patient population with myeloma mirrors that observed in patients with osteoporotic vertebral compression fractures.

These findings complement a 2008 investigation of vertebroplasty in the population with the myeloma by demonstrating the beneficial effects on a much larger patient population and expand these findings by demonstrating that vertebroplasty may confer more favorable outcomes on patients who have already initiated systemic therapy. ${ }^{6}$ Such findings can provide guidance to clinicians and radiologists with respect to the timing of their vertebroplasty therapy and provide additional evidence of the efficacy of spinal augmentation in the palliation of pain and disability as demonstrated in multiple recent studies. ${ }^{11,12}$ The observation that patients on systemic drug therapy have better postoperative pain and disability scores compared with patients not on systemic therapy was unexpected and may suggest that vertebroplasty can be delayed in patients with early disease to optimize the therapeutic response. Indeed, ongoing systemic drug therapy (corticosteroids, chemotherapy, targeted therapy, or biologic therapy) may be advantageous to vertebroplasty by reducing the amount of deformities.

bone turnover and mitigating the amount of bone pain from aberrant osteoblast and osteoclast function. However, it is also possible that the differences in outcomes may be unrelated to systemic therapy and could be a manifestation of an unmeasured confounder or differences in reporting pain among patients with more long-standing bone pain from myeloma. For example, the excess pain reported in the untreated group could reflect additional occult pathologic fractures that are not as common following the initiation of systemic therapy. Furthermore, it is difficult, if not impossible, to extricate myeloma bone pain from mechanical pain in patients not on systemic therapy at the time of vertebroplasty. The origins of this finding merit additional investigation because patients on systemic therapy do experience better outcomes.

The lack of a significant association between the duration of pain and the clinical response to intervention has been previously reported in the vertebroplasty literature but differs somewhat from reports on other spinal procedures (eg, epidural steroid injections) in which patients with longer duration pain have more attenuated benefits to intervention. ${ }^{13-15}$ The discrepancies in these findings may be due to several factors. First, the origin of pain from vertebral compression fractures differs from that of radicular-type pain associated with degenerative disease. Second, patients with radicular-type pain are more likely to have long-standing pain that has failed conservative management, while fracture-related pain is more likely rapidly treated after diagnosis. As such, it is more likely that patients with longterm radicular pain have undergone the physiologic changes associated with chronic pain, with central sensitization and psychological adaptation. ${ }^{16}$ Because the phenomenon of central sensitization has been shown to be related to inflammatory changes in the dorsal root ganglion, such causative mechanisms are not expected to be present in most vertebral compression fracture

This study has several limitations. First, retrospective studies of chronic pain are subject to reporting bias because patients' long-term tolerance to chronic pain can overestimate the significance of a treatment effect, even among newer methods of quantifying chronic pain. While such concerns are valid among longterm pain scores, the observed immediate and short-term improvements in pain and disability are unlikely to be affected by such bias and strongly argue for a favorable treatment effect. Second, our study had substantial loss to follow-up, eliminating $35 \%$ of respondents at the 1-year time point. Such loss to follow-up is 
common among longitudinal studies and can confound results if this loss is a result of unfavorable clinical outcomes following treatment. However, in the case of this study, loss to follow-up was largely due to repeat vertebroplasty treatment or mortality from multiple myeloma. This outcome would argue against the possibility that the lost cohort represented clinical nonresponders. Third, while we observed better outcomes in the patient group already undergoing systemic therapy, the retrospective nature of this study makes it impossible to identify the origins of this observation or ascribe causality. Furthermore, while the observed significant differences between those on systemic therapy and the untreated group suggest that this study is sufficiently powered for these outcomes, sample size limitations prohibit analysis of outcomes for specific systemic therapies. Fourth, selection bias is also likely a confounder in this study because patients who were not referred and/or offered vertebroplasty were not included in this study. It is unknown whether inclusion of these patients, had they undergone vertebroplasty, would have resulted in a similar degree of pain relief.

\section{CONCLUSIONS}

Vertebroplasty provides a safe and effective means of achieving durable pain relief among patients with myeloma with vertebral compression fractures. Outcomes appear to be more favorable among patients who have previously been initiated on systemic drug therapy, yet the reasons for these differences in outcomes remain unclear and should continue to be investigated.

Disclosures: Jennifer S. McDonald-UNRELATED: Grants/Grants Pending: GE Healthcare, ${ }^{*}$ Comments: investigator-initiated research grants for contrast-induced nephropathy studies. David F. Kallmes_UNRELATED: Patents (planned, pending or issued): Mayo Foundation, ${ }^{*}$ Comments: spine augmentation; Royalties: University of Virginia Patent Foundation, Comments: Spine Fusion. Kent R. Thielen-UNRELATED: Royalties: Nevro Corp; Stock/Stock Options: Nevro Corp. Angela DispenzieriUNRELATED: Grants/Grants Pending: Celgene, ${ }^{*}$ Takeda Pharmaceutical Company, ${ }^{*}$ Janssen Biotech, ${ }^{*}$ Pfizer, ${ }^{*}$ Alnylam Pharmaceuticals, ${ }^{*}$ Comments: research dollars to conduct clinical trials. *Money paid to the institution.

\section{REFERENCES}

1. Kallmes DF, Jensen ME. Percutaneous vertebroplasty. Radiology 2003;229:27-36 CrossRef Medline

2. Klazen CA, Lohle PN, de Vries J, et al. Vertebroplasty versus conservative treatment in acute osteoporotic vertebral compression frac- tures (Vertos II): an open-label randomised trial. Lancet 2010;376: 1085-92 CrossRef Medline

3. Kallmes DF, Comstock BA, Heagerty PJ, et al. A randomized trial of vertebroplasty for osteoporotic spinal fractures. N Engl J Med 2009; 361:569-79 CrossRef Medline

4. Cortet B, Cotten A, Boutry N, et al. Percutaneous vertebroplasty in patients with osteolytic metastases or multiple myeloma. Rev Rhum Engl Ed 1997;64:177-83 Medline

5. Layton KF, Thielen KR, Cloft HJ, et al. Acute vertebral compression fractures in patients with multiple myeloma: evaluation of vertebral body edema patterns on MR imaging and the implications for vertebroplasty. AJNR Am J Neuroradiol 2006;27: 1732-34 Medline

6. McDonald RJ, Trout AT, Gray LA, et al. Vertebroplasty in multiple myeloma: outcomes in a large patient series. AJNR Am J Neuroradiol 2008;29:642-48 CrossRef Medline

7. Kyle RA, Gertz MA, Witzig TE, et al. Review of 1027 patients with newly diagnosed multiple myeloma. Mayo Clin Proc 2003;78:21-33 CrossRef Medline

8. Deramond H, Depriester C, Galibert P, et al. Percutaneous vertebroplasty with polymethylmethacrylate: technique, indications, and results. Radiol Clin North Am 1998;36:533-46 CrossRef Medline

9. Patrick DL, Deyo RA, Atlas SJ, et al. Assessing health-related quality of life in patients with sciatica. Spine (Phila Pa 1976) 1995;20:1899908; discussion 909 CrossRef Medline

10. Lauridsen HH, Hartvigsen J, Manniche C, et al. Responsiveness and minimal clinically important difference for pain and disability instruments in low back pain patients. BMC Musculoskelet Disord 2006;7:82 CrossRef Medline

11. Wardlaw D, Cummings SR, Van Meirhaeghe J, et al. Efficacy and safety of balloon kyphoplasty compared with non-surgical care for vertebral compression fracture (FREE): a randomised controlled trial. Lancet 2009;373:1016-24 CrossRef Medline

12. Jha RM, Hirsch AE, Yoo AJ, et al. Palliation of compression fractures in cancer patients by vertebral augmentation: a retrospective analysis. J Neurointerv Surg 2010;2:221-28 CrossRef Medline

13. Alvarez L, Pérez-Higueras A, Granizo JJ, et al. Predictors of outcomes of percutaneous vertebroplasty for osteoporotic vertebral fractures. Spine (Phila Pa 1976) 2005;30:87-92 CrossRef Medline

14. Kaufmann TJ, Geske JR, Murthy NS, et al. Clinical effectiveness of single lumbar transforaminal epidural steroid injections. Pain Med 2013;14:1126-33 CrossRef Medline

15. Rad AE, Kallmes DF. Pain relief following vertebroplasty in patients with and without localizing tenderness on palpation. AJNR Am J Neuroradiol 2008;29:1622-26 CrossRef Medline

16. Latremoliere A, Woolf CJ. Central sensitization: a generator of pain hypersensitivity by central neural plasticity. J Pain 2009;10:895-926 CrossRef Medline 